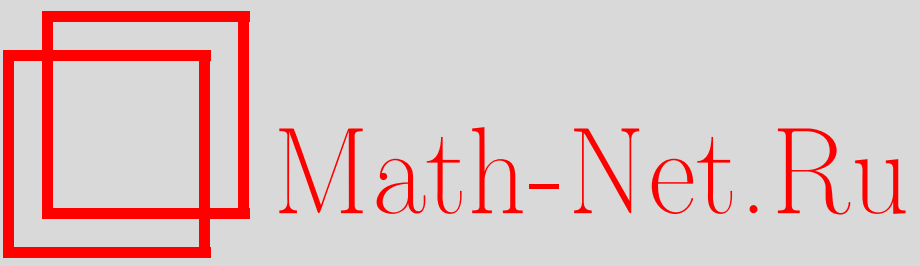

В. А. Кудинов, И. В. Кудинов, Об одном методе получения точного аналитического решения гиперболического уравнения теплопроводности на основе использования ортогональных методов, Вестн. Сам. гос. техн. ун-та. Сер. Физ.-мат. науки, 2010, выпуск 5(), 159-169

DOI: https://doi.org/10.14498/vsgtu804

Использование Общероссийского математического портала Math-Net.Ru подразумевает, что вы прочитали и согласны с пользовательским соглашением http: //www . mathnet.ru/rus/agreement

Параметры загрузки:

IP : 54.89 .56 .158

26 апреля 2023 г., 12:49:48 
УДК $517.958+519.633$

\section{ОБ ОДНОМ МЕТОДЕ ПОЛУЧЕНИЯ ТОЧНОГО \\ АНАЛИТИЧЕСКОГО РЕШЕНИЯ ГИПЕРБОЛИЧЕСКОГО \\ УРАВНЕНИЯ ТЕПЛОПРОВОДНОСТИ НА ОСНОВЕ ИСПОЛЬЗОВАНИЯ ОРТОГОНАЛЬНЫХ МЕТОДОВ}

\section{В.А. Кудинов, И. В. Кудинов}

Самарский государственный технический университет, 443100, Самара, ул. Молодогвардейская, 244.

E-mail: kud-samgtu@yandex.ru

На основе использования метода разделения переменных и ортогонального метода Бубнова-Галёркина получено точное аналитическое решение гиперболического уравнения теплопроводности для бесконечной пластины при граничных условиях первого рода. Показано, что прогрев (охлаждение) тела определяется движением фронта ударной тепловой волны, на котором происходит обрыв температурной кривой (скачок температуръ). Фронт тепловой волнъ разделяет исследуемую область на две подобласти-возмущенную, где температура изменяется от температурь стенки (граничное условие первого рода) до температуры на фронте волны, и невозмущенную, на всем протяжении которой температура равна начальной температуре.

Ключевые слова: гиперболическое уравнение, аналитическое решение, фронт ударной тепловой волны, скорость тепловой волны, время релаксации.

1. Обоснование необходимости применения гиперболических уравнений в краевых задачах. Вывод классического параболического уравнения теплопроводности вида

$$
\frac{\partial t(x, \tau)}{\partial \tau}=a \frac{\partial^{2} t(x, \tau)}{\partial x^{2}}
$$

основывается на гипотезе Фурье о пропорциональности теплового потока градиенту температуры:

$$
q=-\lambda \frac{\partial t}{\partial x}
$$

где $t$ - температура, $x$ - координата; $\tau$ - время; $a$ - коэффициент температуропроводности; $q$ - тепловой поток; $\lambda$ - коэффициент теплопроводности.

В соответствии с (2) изменение температуры в какой-либо точке тела приводит к мгновенному изменению ее во всех других точках тела. Следовательно, в процессе вывода в уравнение (1) закладывается понятие бесконечной скорости распространения теплового возмущения, что подтверждается анализом его аналитических решений. Из этих решений, в частности, следует, что при граничных условиях первого рода (тепловой удар) тепловой поток и скорости движения изотерм при $x \rightarrow 0$ и $\tau \rightarrow 0$ принимают бесконечные значения. Бесконечные значения скоростей движения изотерм отмечаются также вблизи поверхностей, где задаются нулевые граничные условия второго рода (условия адиабатной стенки). Все эти так называемые парадоксы

Василий Александрович Кудинов (д.ф.-м.н., профессор), зав. кафедрой, каф. теоретических основ теплотехники и гидромеханики. Игорь Васильевич Кудинов, аспирант, каф. прикладной математики и информатики. 
теории теплопроводности противоречат фундаментальным физическим законам, ограничивающим бесконечные значения физических величин и скоростей распространения сигналов.

Указанные результаты решений уравнения (1) не отражают действительного (реального) распределения температурных полей. Впервые ещё в XIX веке это было отмечено Риманом, который показал, что различным видам изотермических поверхностей соответствуют различные дифференциальные операторы теплопроводности, и среди них линейный оператор параболического типа встречается лишь как частный случай. И, в частности, Риман дал ответ на вопрос: каким должно быть температурное состояние тела произвольной формы, чтобы система изотерм, заданная в определенный момент времени, оставалась бы системой изотерм в любой момент времени, т. е. чтобы температура любой точки пространства выражалась в виде функции времени и пространственных координат? Кроме того, отвечая на вопрос - какими свойствами должно обладать тело, при которых возможна система кривых, остающихся изотермами, Риман впервые показал, что свойства анизотропного температурного поля характеризуются не теплофизическими параметрами, а видом изотермических поверхностей, описываемых различными дифференциальными операторами, среди которых могут присутствовать операторы, включающие вторую, третью и т. д. производные от искомой функции по пространственным переменным и времени. Отметим, что уравнение диффузии, содержащее высшие производные по координатам и времени, на основе максвелловской теории распределения скоростей молекул было получено А. А. Власовым [1].

Таким образом, параболическому оператору теплопроводности соответствует только строго определенный класс изотермических кривых, выход за пределы которых невозможен путем одного лишь расширения начальных и граничных условий. Поэтому невозможно получить изотермы, несвойственные параболическому оператору, одним лишь изменением этих условий путь, по которому происходило развитие теории теплообмена. Именно этот путь и приводит к перечисленным выше парадоксам и некорректно поставленным задачам, когда решение либо не единственно, либо не существует, либо неустойчиво. Все это связано с ограниченностью параболического оператора теплопроводности, при выводе которого подразумевается, что температура является плавной функцией координат и времени. Однако существуют действительные реальные процессы, имеющие так называемые фронтовые поверхности, при переходе через которые искомая функция и ее производные имеют разрыв. Такие функции описываются, например, гиперболическими дифференциальными операторами.

Таким образом, с одной стороны процесс распространения тепла осуществляется потоком взаимодействующих частиц (электронов, атомов, молекул), что описывается параболическим оператором теплопроводности, а количественные характеристики этого процесса определяются такими свойствами среды, как теплопроводность и теплоемкость. С другой стороны, распространение тепла - волновой процесс, количественными характеристиками которого являются скорость тепловой волны и релаксация теплового потока [2].

Отсюда следует, что в нестационарных процессах закон распределения теплоты, вообще говоря, не подчиняется гипотезе Фурье, что связано с от- 
сутствием в соотношении (2) параметров, учитывающих конечную скорость её распространения. Необходимость введения таких параметров обусловлена тем, что теплота передается мельчайшими частицами вещества (электронами, молекулами, ионными решетками), а конечный результат этой передачи (температура тела) зависит от скоростей движения (колебания) этих частиц. Следовательно, в формуле для распространения теплоты должны учитываться молекулярно-кинетические представления, связанные со скоростями движения частиц, длиной и временем их свободного пробега и взаимодействиями при ударах.

Попытки обобщения уравнения (2) были предприняты авторами работ [2-4], которые независимо друг от друга получили формулу для теплового потока

$$
q=-\lambda \frac{\partial t}{\partial x}-\tau_{r} \frac{\partial q}{\partial \tau}
$$

где $\tau_{r}=a / W^{2}$ - время релаксации (постоянная релаксации); $W$ - скорость продвижения теплового возмущения.

Время релаксации $\tau_{2}$ является характеристикой неравновесности процесса теплопроводности. И оно тем больше, чем больше нарушается тепловое равновесие (тем бо́льшую в этом случае погрешность можно получить при описании процесса уравнением (1)). Отсюда можно заключить, что $\tau_{r}-$ время, необходимое для перевода теплового потока в форму теплового движения частиц, участвующих в процессе передачи теплоты. Следовательно, соотношение $\tau_{r} \partial q / \partial \tau$ в (3) учитывает инерционность теплового потока, т. е. учитывает время, необходимое для его ускорения.

Соотношение (3) принимает вид формулы (2) в случае, если $W \rightarrow \infty$. Так как уравнение 2 положено в основу вывода параболического уравнения (1), то, следовательно, бесконечная скорость распространения теплового возмущения оказывается заложенной в нем уже в процессе вывода.

Отметим, что А. В. Лыковым уравнение вида (3) было получено на основе использования теории Максвелла об аналогии механических свойств жидкостей и твёрдых тел исходя из представления о релаксации - явлении рассасывания упругих напряжений сдвига, т.е. рассеивания упругой энергии, запасенной в деформируемом теле, путем перехода её в теплоту, что связано с хаотическим тепловым движением молекул. На основе этой теории доказывается пропорциональность времени релаксации теплового потока $\tau_{r}$ времени релаксации вязких напряжений.

На основе (3) авторами работ [2-4] было выведено гиперболическое уравнение теплопроводности вида

$$
\frac{\partial t(x, \tau)}{\partial \tau}+\tau_{r} \frac{\partial^{2} t(x, \tau)}{\partial \tau^{2}}=a \frac{\partial^{2} t(x, \tau)}{\partial x^{2}} .
$$

Уравнение (4) было также получено А. С. Предводителевым исходя из совсем иных представлений, а именно из анализа скоростей перемещения изотермических поверхностей, т. е. с использованием представления Римана при полном отказе от релаксационной формулы (3). Факт получения одного и того же уравнения исходя из различных исходных предпосылок позволяет сделать вывод о достаточной физической обоснованности как уравнения (4), так и формулы (3). 
Достоверность уравнения (4) подтверждается также его полной аналогией с телеграфным уравнением, описывающим электрические процессы. Тождественность переноса тепла и электричества базируется на аналогии законов Фурье и Ома, подтверждающейся законом Видемана-Франца, согласно которому отношение коэффициентов теплопроводности и электропроводности для всех металлов при одной и той же температуре одинаково. Следовательно, электроны проводимости переносят не только электрический заряд, но и теплоту. В металлах, где концентрация свободных электронов высока, практически вся теплота переносится электронами, в связи с чем металлы с высокой электропроводностью являются также и высокотеплопроводными.

О наличии аналогии между этими двумя явлениями свидетельствует также тот факт, что переход вещества в состояние сверхпроводимости (резкое падение электрического сопротивления) при низких температурах сопровождается резким увеличением теплопроводности. Например, коэффициент теплопроводности жидкого гелия при температуре $-270{ }^{\circ} \mathrm{C}$ составляет $\lambda=$ $=0,0106(\mathrm{Bт} / \mathrm{m} \cdot \mathrm{K})$, а при температуре $-271^{\circ} \mathrm{C}$ он увеличивается до величины $\lambda=0,9869 \cdot 10^{6} \mathrm{BT} /(\mathrm{m} \cdot \mathrm{K})$, т. е. явление сверхпроводимости сопровождается также явлением сверхтеплопроводности. Время релаксации теплового потока в данном случае составляет $\tau_{r} \approx 10^{-3}$ с, а скорость продвижения тепловой волны $W=19 \mathrm{~m} /$ с [2]. Такие величины $\tau_{r}$ и $W$ приводят к соизмеримости второго слагаемого левой части уравнения (4) с другими его членами и, следовательно, для определения температуры необходимо привлекать гиперболическое уравнение теплопроводности.

Для большинства практических задач теплопроводности влияние конечной скорости распространения теплоты в уравнении (4) пренебрежимо мало. В самом деле, скорость диффузного распространения теплоты на 10 порядков меньше скорости движения фронта тепловой волны [5]. Однако в ряде тепловых процессов наиболее достоверный результат можно получить лишь на основе исследования решений гиперболических уравнений теплопроводности. K их числу относятся высокоинтенсивные нестационарные процессы, время протекания которых сопоставимо с временем релаксации $\tau_{r}$, например, при нагреве металлов короткими лазерными импульсами (длительностью от нано- до фемтосекунд). Скорость нагревания здесь сопоставима с временем термализации, которое необходимо электронам для обмена энергией с решеткой, и с временем релаксации, которое нужно для изменения их состояния. $\mathrm{K}$ числу других относятся процессы нагревания при трении с высокой скоростью, при анализе физических механизмов возникновения теплового удара, локального нагрева при динамическом распространении трещины в околозвуковом режиме и т. п. [6].

Из решения уравнения (4) для малых значений временно́й и пространственной координат $(\tau \rightarrow 0, x \rightarrow 0)$ следуют выводы, противоположные тем, которые получаются из решения уравнения (1). И, в частности, тепловой поток на стенке и скорости движения изотерм при $x \rightarrow 0$ и $\tau \rightarrow 0$ принимают уже не бесконечные, а нулевые значения, что вполне объясняется инерционным характером распространения теплоты с учетом конечной скорости этого процесса. Следует, однако, отметить, что при больших значениях времени и пространственной координаты решения уравнений (1) и (4) для определенного круга задач (см. ниже) полностью совпадают. В связи с этим возникает 
вопрос: в каких диапазонах временных интервалов необходимо использовать решения гиперболического уравнения, а в каких, не допуская значительных погрешностей, можно пренебречь конечной скоростью распространения теплоты?

Для ответа на поставленные выше вопросы необходимо выполнить детальное исследование точного аналитического решения гиперболического уравнения теплопроводности для конечного пространства. В известной литературе решения этого уравнения приводятся в основном лишь для полупространства, что затрудняет их исследование ввиду трудностей представления исходной математической постановки задачи полностью в безразмерном виде (для всех переменных задачи) и не позволяет выполнить детальный анализ и сделать заключения наиболее общего вида. Кроме того, решение для полупространства не позволяет выполнить исследование обратной тепловой волны, возникающей при достижении фронтом теплового возмущения противоположной стенки. На фронте обратной тепловой волны, так же как и на фронте прямой волны, наблюдается обрыв температурных кривых.

2. Математическая постановка задачи. В настоящей работе получено точное аналитическое решение гиперболического уравнения теплопроводности для бесконечной пластины при симметричных граничных условиях первого рода. Математическая постановка задачи имеет вид:

$$
\begin{aligned}
& \frac{\partial t(x, \tau)}{\partial \tau}+\tau_{r} \frac{\partial^{2} t(x, \tau)}{\partial \tau^{2}}=a \frac{\partial^{2} t(x, \tau)}{\partial x^{2}} \quad(\tau>0,0 \leqslant x \leqslant \delta) \\
& t(x, 0)=t_{0} ; \quad \frac{\partial t(x, 0)}{\partial \tau}=0 ; \quad \frac{\partial t(0, t)}{\partial x}=0 ; \quad t(\delta, \tau)=t_{\mathrm{cT}}
\end{aligned}
$$

где $t_{0}$ - начальная температура; $t_{\text {ст }}$ - температура стенки; $\delta$ - толщина пластины.

Введём следующие обозначения:

$$
\Theta=\left(t-t_{\mathrm{cT}}\right) /\left(t_{0}-t_{\mathrm{cT}}\right), \quad \xi=x / \delta, \quad \text { Fо }=a \tau / \delta^{2},
$$

где $\Theta$ - относительная избыточная температура; $\xi$ - безразмерная координата; Fо - число Фурье.

С учётом принятых обозначений задача (5), (6) запишется так:

$$
\begin{gathered}
\frac{\partial \Theta(\xi, \mathrm{Fo})}{\partial \mathrm{Fo}}+\mathrm{Fo}_{\mathrm{r}} \frac{\partial^{2} \Theta(\xi, \mathrm{Fo})}{\partial \mathrm{Fo}^{2}}=\frac{\partial^{2} \Theta(\xi, \mathrm{Fo})}{\partial \xi^{2}} \\
(\mathrm{Fo}>0,0 \leqslant \Theta \leqslant 1,0 \leqslant \xi \leqslant 1) \\
\Theta(\xi, 0)=1 ; \\
\partial \Theta(\xi, 0) / \partial \mathrm{Fo}=0 \\
\partial \Theta(0, \mathrm{Fo}) / \partial \xi=0 \\
\Theta(1, \mathrm{Fo})=0
\end{gathered}
$$

где $\mathrm{Fo}_{\mathrm{r}}=a \tau_{r} / \delta^{2}$.

3. Последовательность решения задачи. Решение задачи (8)-(12) принимается в виде

$$
\Theta(\xi, \mathrm{Fo})=\varphi(\mathrm{Fo}) \psi(\xi)
$$


Подставляя (13) в (8), находим

$$
\begin{gathered}
\mathrm{F}_{\mathrm{r}} \frac{d^{2} \varphi}{d \mathrm{Fo}^{2}}+\frac{d \varphi}{d \mathrm{Fo}}+\nu \varphi=0 \\
\frac{d^{2} \psi}{d \xi^{2}}+\nu \psi=0
\end{gathered}
$$

где $\nu=\mu \mathrm{Fo}_{\mathrm{r}}, \mu$ - некоторая постоянная.

Граничные условия для уравнения Штурма-Лиувилля (15) исходя из (11) и (12) задаются соотношениями

$$
\begin{gathered}
d \psi(0) / d \xi=0 \\
\psi(1)=0
\end{gathered}
$$

Решение уравнения (15) с граничными условиями (16), (17) принимается в виде [7]

$$
\psi(\xi)=\cos \left(r \frac{\pi}{2} \xi\right), \quad r=2 k-1, k \in \mathbb{N} .
$$

Соотношение (18) удовлетворяет граничным условиям (16), (17). Для нахождения собственных чисел составим невязку уравнения (15) и потребуем ортогональности невязки к функциям $\cos (r \pi \xi / 2)(r=2 k-1, k \in \mathbb{N})$, т. е.

$$
\int_{0}^{1}\left[-r^{2} \frac{\pi^{2}}{4} \cos \left(r \frac{\pi}{2} \xi\right)+\nu \cos \left(r \frac{\pi}{2} \xi\right)\right] \cos \left(j \frac{\pi}{2} \xi\right) d \xi=0,
$$

где $j=r=2 k-1, k \in \mathbb{N}$.

Ввиду ортогональности косинусов соотношение (19) примет вид

$$
\int_{0}^{1}\left[-r^{2} \frac{\pi^{2}}{4} \cos ^{2}\left(r \frac{\pi}{2} \xi\right)+\nu \cos ^{2}\left(r \frac{\pi}{2} \xi\right)\right] d \xi=0
$$

Вычисляя интегралы в (20), получаем

$$
\nu_{k}=\frac{1}{4} r^{2} \pi^{2}, \quad r=2 k-1, k \in \mathbb{N} .
$$

Собственные числа, найденные по формуле (19)), полностью совпадают с точными их значениями [3]. Собственные функции для каждого собственного числа находятся из (18). Нетрудно убедиться, что соотношение (18) при использовании собственных чисел, определяемых по формуле (21), точно удовлетворяет уравнению Штурма-Лиувилля (15). В самом деле, соотношение $r \pi / 2$ аргумента косинуса можно представить как $r \pi / 2=\sqrt{\nu_{k}}, r=2 k-1$, $k \in \mathbb{N}$, следовательно, оно представляет собой спектр собственных чисел $[7,8]$.

Характеристическое уравнение для однородного дифференциального уравнения второго порядка (14) имеет вид

$$
\mathrm{Fo}_{\mathbf{r}} z^{2}+z+\nu_{k}=0
$$

Уравнение (22) для каждого собственного числа $\nu_{k}$ имеет два действительных отрицательных корня $z_{1 k}, z_{2 k}$ :

$$
z_{i k}=\left(-1 \pm \sqrt{1-4 \mathrm{Fo}_{\mathrm{r}} \nu_{k}}\right) /\left(2 \mathrm{Fo}_{\mathrm{r}}\right), \quad i \in\{1,2\}, k \in \mathbb{N} .
$$


С учётом найденных значений $z_{1 k}$ и $z_{2 k}$ решение уравнения (22) для каждого собственного числа примет вид

$$
\varphi_{k}(\mathrm{Fo})=C_{1 k} \exp \left(z_{1 k} \mathrm{Fo}\right)+C_{2 k} \exp \left(z_{2 k} \mathrm{Fo}\right)
$$

где $C_{i k}$ - неизвестные коэффициенты; $i \in\{1,2\}, k \in \mathbb{N}$.

Подставляя (18), (23) в (13), находим

$$
\Theta_{k}(\xi, \mathrm{Fo})=\left[C_{1 k} \exp \left(z_{1 k} \mathrm{Fo}\right)+C_{2 k} \exp \left(z_{2 k} \mathrm{Fo}\right)\right] \cos \left(r \frac{\pi}{2} \xi\right)
$$

где $r=2 k-1 ; k \in \mathbb{N}$.

Каждое частное решение (24) точно удовлетворяет уравнению (8) и граничным условиям (11), (12), но ни одно из них, в том числе и их сумма

$$
\begin{gathered}
\Theta(\xi, \mathrm{Fo})=\sum_{k=1}^{\infty}\left\{\left[C_{1 k} \exp \left(z_{1 k} \mathrm{Fo}\right)+C_{2 k} \exp \left(z_{2 k} \mathrm{Fo}\right)\right] \cos \left(r \frac{\pi}{2} \xi\right)\right\}, \\
r=2 k-1, \quad k \in \mathbb{N}
\end{gathered}
$$

не удовлетворяют начальным условиям (9), (10). Для выполнения начальных условий составляет их невязка и требуется ортогональность невязки ко всем собственным функциям $\cos (r \pi \xi / 2)$ :

$$
\begin{aligned}
& \int_{0}^{1}\left[\sum_{k=1}^{\infty}\left(C_{1 k} e^{z_{1 k} \mathrm{Fo}}+C_{2 k} e^{z_{2 k} \mathrm{~F} \circ}\right) \cos \left(r \frac{\pi}{2} \xi\right)-1\right]_{\mathrm{Fo}=0} \cos \left(j \frac{\pi}{2} \xi\right) d \xi=0, \\
& \int_{0}^{1}\left[\sum_{k=1}^{\infty}\left(C_{1 k} z_{1 k} e^{z_{1 k} \mathrm{Fo}}+C_{2 k} z_{2 k} e^{z_{2 k} \mathrm{~F} \circ}\right) \cos \left(r \frac{\pi}{2} \xi\right)\right]_{\mathrm{F}=0} \cos \left(j \frac{\pi}{2} \xi\right) d \xi=0,
\end{aligned}
$$

где $j=r=2 k-1, k \in \mathbb{N}$. Последние соотношения можно переписать следующим образом:

$$
\begin{aligned}
& \int_{0}^{1}\left[\sum_{k=1}^{\infty}\left(C_{1 k}+C_{2 k}\right) \cos \left(r \frac{\pi}{2} \xi\right)-1\right] \cos \left(j \frac{\pi}{2} \xi\right) d \xi=0 \\
& \int_{0}^{1}\left[\sum_{k=1}^{\infty}\left(C_{1 k} z_{1 k}+C_{2 k} z_{2 k}\right) \cos \left(r \frac{\pi}{2} \xi\right)\right] \cos \left(j \frac{\pi}{2} \xi\right) d \xi=0
\end{aligned}
$$

Соотношения (26), (27) ввиду ортогональности косинусов примут вид

$$
\begin{gathered}
\int_{0}^{1}\left(C_{1 k}+C_{2 k}\right) \cos ^{2}\left(r \frac{\pi}{2} \xi\right) d \xi-\int_{0}^{1} \cos \left(r \frac{\pi}{2} \xi\right) d \xi=0 \\
\int_{0}^{1}\left(C_{1 k} z_{1 k}+C_{2 k} z_{2 k}\right) \cos ^{2}\left(r \frac{\pi}{2} \xi\right) d \xi=0 .
\end{gathered}
$$

где $r=2 k-1, k \in \mathbb{N}$. 
Вычисляя интегралы в соотношениях (28), (29), относительно неизвестных коэффициентов $C_{1 k}, C_{2 k}$ получаем систему двух алгебраических линейных уравнений. Её решение

$$
C_{1 k}=-C_{2 k} \frac{z_{2 k}}{z_{1 k}}, \quad C_{2 k}= \pm \frac{4}{r \pi}\left(1-\frac{z_{2 k}}{z_{1 k}}\right)^{-1},
$$

где знак «плюс» берётся при $r=4 k-3$, знак «минус»- при $r=4 k-1, k \in \mathbb{N}$.

После определения неизвестных коэффициентов $C_{1 k}$ и $C_{2 k}$ точное аналитическое решение задачи (8)-(12) находится из (25). Непосредственной подстановкой можно убедиться, что все исходные уравнения задачи (8)-(12) удовлетворяются точно.

4. Анализ полученных результатов. На рис. 1, 2 приведены результаты расчётов безразмерной температуры по формуле (25) для следующих исходных данных задачи: $a=10^{-6} \mathrm{~m}^{2} / \mathrm{c} ; \tau_{r}=10^{-7} \mathrm{c} ; \delta=0,001 \mathrm{~m}$.

Анализ результатов позволяет сделать вывод о том, что при малых значениях времени $\mathrm{Fo}$ и пространственной координаты $\xi$ на фронте температурного возмущения наблюдается обрыв температурных кривых, т. е., по существу, образуется фронт ударной тепловой волны, на границе которого наблюдается скачок температуры от величины температуры в точке обрыва до начальной температуры. Область тела, находящаяся за пределами фронта тепловой волны, оказывается невозмущённой, и температура здесь равна начальной температуре. Такой результат является следствием конечной скорости распространения теплоты, которая для условий данной конкретной задачи составит величину $W=\sqrt{a / \tau_{r}}=3,16228 \mathrm{~m} / \mathrm{c}$.

Температура в точках обрыва описывается формулой

$$
t(\tau)=t_{0}-\exp \left(-\tau /\left(2 \tau_{r}\right)\right)
$$

полностью совпадающей с соотношением для температуры в точках обрыва, полученным в работах $[4,5]$, где решения были найдены для полупространства.

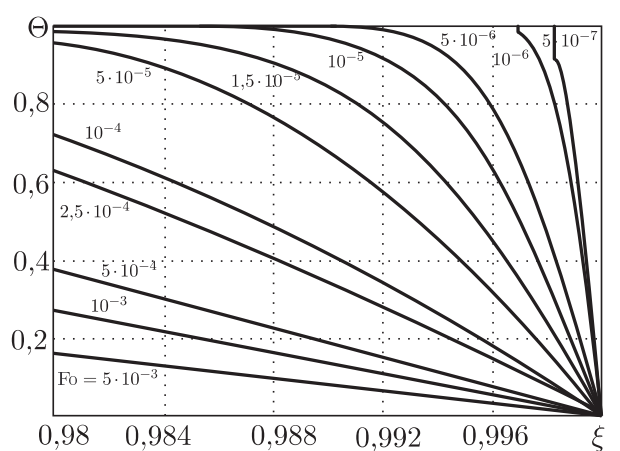

Рис. 1. Изменение температуры в пластине с учётом конечной скорости распространения теплоты; $5 \cdot 10^{-7} \leqslant$ Fo $\leqslant 5 \cdot 10^{-3}$, $\mathrm{Fo}_{\mathrm{r}}=10^{-7}$

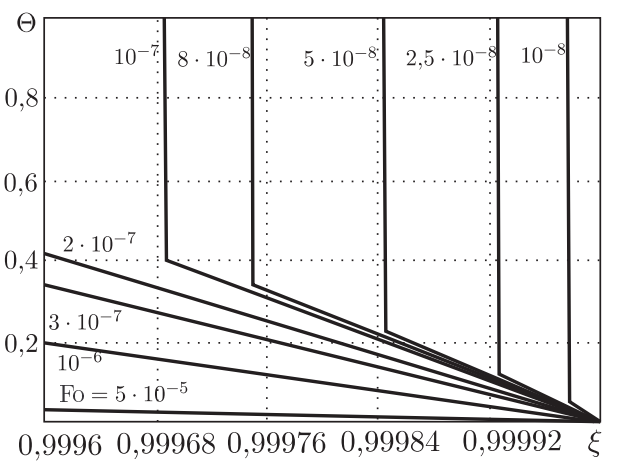

Рис. 2. Изменение температуры в пластине с учётом конечной скорости распространения теплоты; $10^{-8} \leqslant \mathrm{Fo} \leqslant 5 \cdot 10^{-5}$,

$$
\mathrm{Fo}_{\mathrm{r}}=10^{-7}
$$


Отмечается линейная закономерность движения точки обрыва по пространственной координате во времени $x=\delta-W \tau$ (рис. 3). Этот факт подтверждается исследованиями, приведёнными в [5].

После достижения в точке обрыва температуры $\Theta=1,0$ (для приведённых выше исходных данных задачи это происходит при $\mathrm{Fo} \approx 6 \cdot 10^{-6}$ ) полученные по формуле (25) результаты полностью совпадают с решением параболического уравнения теплопроводности (см. рис. 1).

Ответ на поставленный выше вопрос о разграничении временных интервалов использования решений параболического и гиперболического уравнений можно получить на основе исследования решения гиперболического уравнения теплопроводности при различных значениях числа $\mathrm{Fo}_{\mathrm{r}}$. Эти исследования показали, что с увеличением $\mathrm{Fo}_{\mathrm{r}}$ обрыв температурных кривых наблюдается для бо́льших значений числа Fo и координаты $\xi$. Так, например, для приведенных выше исходных данных $\mathrm{Fo}_{\mathrm{r}}=10^{-7}$. Обрыв температурных кривых здесь заканчивается при $\mathrm{Fo} \approx 6 \cdot 10^{-6}$. Расстояние, на которое проникает фронт ударной тепловой волны по координате $\xi$, в этом случае составляет $1-\xi \approx 0,008$. В случае, если $\mathrm{Fo}_{\mathrm{r}}=6,25 \cdot 10^{-3}$, окончание обрыва температурных кривых наблюдается при $\mathrm{Fo} \approx 0,08$. Температурные возмущения для этого $F o$ распространяются практически на всю толщину пластины - координата фронта возмущения составляет $1-\xi \approx 0,94$ (см. рис. 4). Для $\mathrm{Fo}_{\mathrm{r}}=0,1$ обрыв температурных кривых не заканчивается при достижении фронтом температурного возмущения координаты $1-\xi \approx 1$. В этом случае температура на стенке при $\xi \approx 0$ (адиабатная стенка) при Fo $\approx 0,31623$ скачком изменяется от температуры $\Theta=1,0$ до $\Theta \approx 0,79$. При дальнейшем охлаждении тела возникает обратная тепловая волна, на фронте которой также наблюдается обрыв температурных кривых.

Полученные результаты позволяют сделать вывод, что во всех случаях, когда время релаксации $\tau_{r}$ велико, а скорость движения фронта ударной тепловой волны $W$ мала, необходимо использовать гиперболическое уравнение теплопроводности. При этом исследуемые процессы не обязательно должны быть быстропротекающими.

Следует отметить, что сходимость ряда (25) существенно зависит от величины числа Фурье, для которого необходимо определять распределение температуры по толщине пластины. Например, для задачи с приведенными

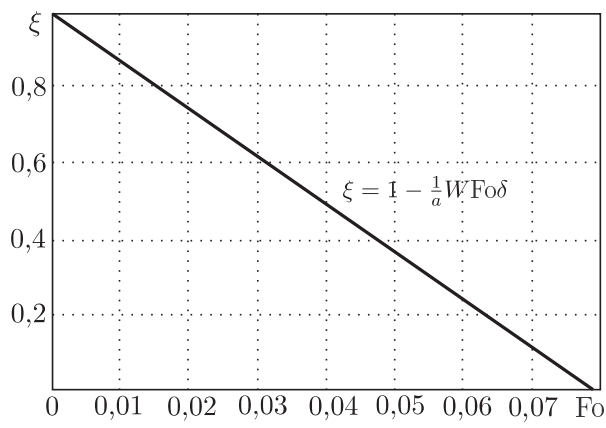

Рис. 3. Перемещение фронта ударной тепловой волны по координате $\xi$ во времени Fo; $\mathrm{Fo}_{\mathrm{r}}=6,25 \cdot 10^{-3}$

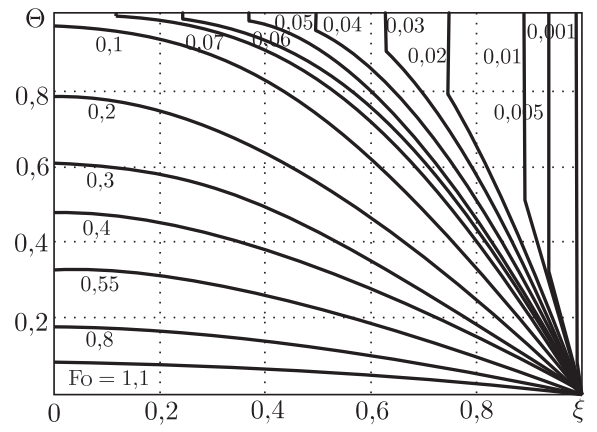

Рис. 4. Распределение температуры в пластине с учётом конечной скорости распространения теплоты; $\mathrm{Fo}_{\mathrm{r}}=6,25 \cdot 10^{-3}$ 
выше исходными данными в диапазоне числа Фурье $0,1 \leqslant$ Fo $<\infty$ для сходимости достаточно всего нескольких членов этого ряда. При $0,0001 \leqslant \mathrm{Fo} \leqslant 0,1$ сходимость имеет место при 10-1000 членах ряда. Для всех чисел Фурье, при которых происходит обрыв температурных кривых, число членов ряда (25), необходимых для его сходимости, существенно возрастает (от 10000 при $\mathrm{Fo}=10^{-5}$ до 1000000 при $\left.\mathrm{Fo}=10^{-8}\right)$. При дальнейшем уменьшении числа Фурье количество членов ряда (25) может достигать нескольких миллионов. Для расчётов таких рядов требуются компьютеры с большим быстродействием. Максимальное число членов ряда, использованных в настоящей работе, равнялось 2000000 (для $\mathrm{Fo}=10^{-9}$ ). При этом время работы компьютера на базе Inte1 ${ }^{\odot}$ Core $^{\text {TM }} 2$ Cuad CPU Q9400 2,66 ГГц с 3,25 Гб ОЗУ составляло около 8 часов. Отметим, что сходимость ряда (25) в данном случае не была достигнута. В связи с этим кривая изменения температуры для $\mathrm{Fo}=10^{-9}$ на графиках представленных рисунков не приведена.

\section{БИБЛИОГРАФИЧЕСКИЙ СПИСОК}

1. Власов A. А. Статистические функции распределения. - М.: Наука, 1966. - 355 с.

2. Шашков А.Г., Бубнов. В.А., Яновский С.Ю. Волновые явления теплопроводности: системно-структурный подход. - М.: Едиториал УРСС, 2004. - 296 с.

3. Лыков A. В. Теория теплопроводности. - М.: Высш. шк., 1967. -600 с.

4. Лыков А. В. Тепломассоперенос. - М.: Энергия, 1971. - 560 с.

5. Баумейстер K., Хатилл T. Гиперболическое уравнение теплопроводности. Решение задачи о полубесконечном теле // Теплопередача, 1969. - № 4. - С. 112-119.

6. Жоу Д., Касас-Баскес Х., Лебон Д. Ж. Расширенная необратимая термодинамика. М.-Ижевск: РХД, 2006. - 528 с.

7. Кудинов В.А., Карташов Э.М., Калашников В. В. Аналитические решения задач тепломассопереноса и термоупругости для многослойных конструкций. - М.: Высш. шк., 2005. $-430 \mathrm{c}$.

8. Кудинов В.А., Аверин Б. В., Стефанюк Е. В. Теплопроводность и термоупругость в многослойных конструкциях. - М.: Высш. шк., 2008. - 391 с.

Поступила в редакцию 28/VI/2010;

в окончательном варианте - 10/IX/2010. 
MSC: 80A17, 80M25

\section{ABOUT ONE METHOD OF OBTAINING OF THE EXACT ANALYTICAL DECISION OF THE HYPERBOLIC EQUATION OF HEAT CONDUCTIVITY ON THE BASIS OF USE OF ORTHOGONAL METHODS}

\section{A. Kudinov, I. V. Kudinov}

Samara State Technical University,

244, Molodogvardeyskaya st., Samara, 443100, Russia.

E-mail: kud-samgtu@yandex.ru

On the basis of use of a method of division of variables and an orthogonal method of Bubnov-Galyorkin the exact analytical decision of the hyperbolic equation of heat conductivity for an infinite plate under boundary conditions of the first sort is obtained. It is shown that having warmed up (or cooled)a body it is defined by movement of front of a shock thermal wave on which there is a breakage temperature curve (temperature jump). The front of a thermal wave divides investigated area on two subareas - revolted where the temperature changes from wall temperature (a boundary condition of the first sort) to the temperature at the front waves and not revolted where the temperature is equal to reference temperature.

Key words: hyperbolic equation, analytical decision, shock thermal wave front, thermal wave speed, relaxation time.

Original article submitted 28/VI/2010; revision submitted 10/IX/2010.

Vasily A. Kudinov (Dr. Sci. (Phys. \& Math.)), Head of Dept., Dept. of Theoretical Basis of Heat Engineering \& Flow Mechanics. Igor V. Kudinov, Postgraduate Student, Dept. of Applied Mathematics \& Computer Science. 